Suárez Rincón, M.L. (2018). Estrategias pedagógicas para la enseñanza de las matemáticas en Administración: Estudios y experiencias. Revista Electrónica Interuniversitaria de Formación del Profesorado, 21(2), 79-89.

DOI: http://dx.doi.org/10.6018/reifop.21.2.323261

\title{
Estrategias pedagógicas para la enseñanza de las matemáticas en Administración: Estudios y experiencias
}

Martha Luz Suárez Rincón

Colegio de Estudios Superiores de Administración CESA

\section{Resumen}

Este escrito recoge experiencias de diferentes universidades de Hispanoamérica en torno a la problemática de la enseñanza y el aprendizaje de las matemáticas en estudios de pregrado en Administración. Los estudios reseñados, así como las acciones que se vienen implementando en el Colegio de Estudios Superiores de Administración, se presentan desde las dimensiones de los estudiantes, los profesores y el currículo.

\section{Palabras clave}

Aprendizaje basado en problemas; Aprendizaje activo; Didáctica de las matemáticas; Matemáticas en Administración

\section{Pedagogical strategies for the teaching of mathematics in Administration: Studies and experiences}

\begin{abstract}
This paper gathers experiences of different universities in Latin America about the problems of teaching and learning mathematics in Business undergraduate studies. The reviewed studies, as well as the actions that are being implemented in CESA, are presented from three dimensions: students, teachers and curriculum.
\end{abstract}

\section{Key words}

Problem-Based learning; Active Learning; Math didactics; Math in Business

\section{Contacto:}

Martha Luz Suárez Rincón, mluzsuarez@cesa.edu.co, Docente de Carrera Académica, Área de matemáticas y estadística. Colegio de Estudios Superiores de Administración, CESA. Bogotá, Colombia. 


\section{Introducción}

La enseñanza de las matemáticas en general, tanto en niveles básicos como universitarios, plantea siempre un desafío para los docentes en la búsqueda de estrategias pedagógicas y alternativas didácticas que promuevan eficazmente el aprendizaje en los estudiantes. El presente escrito pretende, por una parte, reseñar experiencias recientes de investigación realizadas en Hispanoamérica centradas en la enseñanza y el aprendizaje de matemáticas en programas de Administración; por otra parte, presentar las estrategias y actividades de aprendizaje que se vienen desarrollando en el Colegio de Estudios Superiores de Administración con el objetivo de promover el aprendizaje de matemáticas en sus estudiantes. No se pretende presentar resultados de investigación, pues en el presente en el Cesa el equipo de profesores está en una etapa de exploración de metodologías que potencien el aprendizaje, entre ellas el aprendizaje basado en proyectos. En un futuro cercano se espera sistematizar las experiencias de aula y formular las hipótesis y preguntas que den lugar a un proyecto completo de investigación.

Existen factores de muy diversa índole que están presentes a la hora de planear, desarrollar y evaluar una clase de matemáticas. Dichos factores impactan el logro de los resultados de aprendizaje de los estudiantes en mayor o menor medida y vale la pena reflexionar acerca de ellos, vinculándolos con las propuestas innovadoras que han desarrollado docentes universitarios hispanoamericanos en torno a la pedagogía de las matemáticas en educación superior, particularmente en pregrados de Administración.

Esta reflexión en torno a los factores relacionados con el aprendizaje de las matemáticas en programas de administración tiene antecedentes como el estudio realizado en la Universidad del Zulia donde "se aborda a partir de una revisión documental sobre la enseñanza de las matemáticas y en el proceso de inferencia sobre los resultados obtenidos con los problemas planteados" (García de Pilo, 2010). En este estudio la autora realiza un análisis sobre los factores relacionados con el estudiante, la infraestructura, el profesor y el currículo. Esto la lleva a concluir sobre la necesidad de tener en cuenta para el diseño instruccional de las clases de matemáticas la inclusión de reglas claras, la promoción del autoaprendizaje en los estudiantes, realizar algunos cambios en la actitud de los docentes y fortalecer el trabajo en equipo. (García de Pilo, 2010)

El punto de partida que se elige en el presente documento para identificar y caracterizar los factores involucrados en la pedagogía de las matemáticas es la tríada estudiante - docente - currículo, como se desarrolla a continuación.

\section{Factores a considerar}

\section{Estudiantes}

Como principales actores del proceso de enseñanza aprendizaje, el imaginario creado entre los estudiantes en torno al estudio de las matemáticas cobra gran relevancia. Para los estudiantes, el estudio del álgebra y el cálculo tiene una connotación de dificultad elevada que a veces determina la elección de su carrera universitaria según la cantidad de matemáticas que presente el currículo. También hay un cierto escepticismo entre los estudiantes acerca de la aplicabilidad y utilidad en la vida profesional de los conceptos presentes en estas disciplinas, lo cual influye directamente en la baja motivación hacia el aprendizaje del cálculo y el álgebra. De allí que diferentes autores hayan realizado trabajos para definir, medir y relacionar las actitudes de los estudiantes frente a las matemáticas. 
¿Qué se entiende por actitud hacia las matemáticas? Algunos autores la definen como "una serie de disposiciones que manifiesta el individuo para aceptar o no, familiarizarse o no, con determinados contenidos matemáticos." (Petriz, Barona, López, \& Quiroz, 2010)

Existe literatura abundante en torno a la relación de las actitudes frente a las matemáticas de los estudiantes y su rendimiento académico. Se destacan propuestas como la Escala de Actitudes frente a las Matemáticas (Auzmendi, 1992) que han sido aplicadas y referenciadas en otros estudios (Dorfer \& Ulloa, 2016) (Rojas, 2017) (Zubieta \& Martínez, 2008) (Veliz \& Pérez, 2004).

Los estudios coinciden en la percepción de las matemáticas por parte de los estudiantes como una asignatura difícil de aprender, causante de ansiedad y frustración, aunque reconozcan la necesidad de aprenderlas aun sin tener claro qué tanto las aplicarán en su vida profesional. Por otra parte, sí se ha encontrado relación entre las actitudes frente a las matemáticas y los resultados académicos, pues los estudiantes que manifiestan agrado o encuentran utilidad al aprendizaje de conceptos matemáticos tienen mejores desempeños. (Dorfer \& Ulloa, 2016) (Veliz \& Pérez, 2004)

Otro factor a considerar está relacionado con los estilos de aprendizaje, el cual fue abordado en un estudio con estudiantes de primer semestre de la Universidad de Luján. De acuerdo con los autores Alonso, Gallego y Honey (citados en Cagliolo, Junco \& Peccia, 2010):" Los Estilos de Aprendizaje son los rasgos cognitivos, afectivos y fisiológicos, que sirven como indicadores relativamente estables, de cómo los discentes perciben, interaccionan y responden a sus ambientes de aprendizaje". En este estudio, se encontró que los estudiantes con estilos de aprendizaje activo y teórico obtuvieron resultados superiores en matemáticas. (Cagliolo, Junco, \& Peccia, 2010)

Por otra parte, otros estudios han evidenciado el bajo desarrollo del pensamiento abstracto en los estudiantes universitarios. El pensamiento abstracto “... constituye el nivel superior del pensamiento lógico-matemático. La incapacidad de muchos estudiantes universitarios para enfrentarse al pensamiento lógico matemático formal podría deberse a que no han logrado el nivel de desarrollo cognitivo apropiado." (Hernández, Ramírez, \& Rincón, 2013).

En el caso las de carreras de pregrado relacionadas con Administración, las asignaturas de matemáticas son parte de los primeros tres semestres y no son percibidas por los estudiantes como parte del núcleo profesional. Esto hace bajar la motivación frente al estudio de las matemáticas, pues no se tiene tan clara la aplicación de conceptos del cálculo diferencial o integral en la vida profesional. La motivación, así como la autoeficacia entendida como la creencia en la propia capacidad de organizar y ejecutar los cursos de acción necesarios para gestionar las situaciones posibles (Bandura, 1997)- se constituyen como otros factores relacionados con el aprendizaje de las matemáticas.

En el trabajo de tesis doctoral de Flor de María Camposeco (2012), se indica que:

La autoeficacia y motivación de los estudiantes fue predictor importante en sus logros en matemáticas. La influencia de la autoeficacia es importante porque predice no solo el desempeño en matemáticas de los estudiantes, sino también otras variables que influyen en aspectos sobre todo el logro en matemáticas. (Camposeco, 2012)

Uno de los hallazgos de este trabajo, adelantado con estudiantes entre los 17 y 21 años, tanto españoles como latinoamericanos, tiene que ver con la percepción que tienen los estudiantes acerca de su propia capacidad frente a las matemáticas y cómo se relaciona con la motivación intrínseca o extrínseca. Los que tenían un autoconcepto alto sobre sus capacidades matemáticas, reflejaban una mayor motivación intrínseca y estaban dispuestos 
a aceptar mayores retos y desafíos. Mientras tanto, los que tenían autoconceptos más bajos, desarrollaban mayor motivación extrínseca, orientada a cumplir con aprobar sus asignaturas, sólo cuando era obligatorio para ellos. (Camposeco, 2012)

En resumen, puede afirmarse que los factores que influyen en el aprendizaje de matemáticas en estudiantes de primeros semestres de administración son: las actitudes que tienen frente a las matemáticas, su motivación y autoeficacia, sus estilos de aprendizaje y el desarrollo de pensamiento abstracto que traen desde la escuela básica.

\section{Profesores}

Los profesores juegan un rol protagónico al ser los mediadores en el proceso de enseñanza aprendizaje. El profesor debe resolver las situaciones profesionales y los problemas del salón de clase desde la dificultad de la inmediatez. (Contreras Domingo, 1994)

Los profesores son quienes tienen la responsabilidad y el compromiso de estar enfocados en el aprendizaje de los estudiantes. Quienes llegan a ser docentes en las facultades de Administración cuentan con formación disciplinar diversa, porque no sólo tienen títulos de pregrado en matemáticas sino también en otros campos como la Ingeniería. No es tan fácil para los profesores desprenderse de los sesgos que traen de su propia formación, los cuales suelen transferir a su práctica docente. Es importante señalar que se ha venido trabajando en la formación de docentes en las instituciones para fortalecer aspectos relacionados con la pedagogía y la didáctica. Podría decirse que estamos en una etapa de tránsito hacia nuevas orientaciones pedagógicas y metodológicas en la enseñanza de las matemáticas.

Hay trabajos centrados en el análisis de las didácticas en la enseñanza de las matemáticas en los que se reflexiona sobre la organización de conocimientos que prepara el profesor para facilitar su comprensión por parte de los estudiantes. (Silva Atacho, 2012)

Se han realizado también trabajos en relación con la formación del profesorado para promover el pensamiento crítico y la resolución de problemas, de forma tal que los profesores de matemáticas lo puedan integrar a sus prácticas docentes y favorecer el aprendizaje en sus estudiantes. (Vargas, 2011)

Lo anterior conduce al papel que los docentes juegan en la búsqueda de estrategias pedagógicas que promuevan en sus estudiantes actitudes más positivas, que reconozcan los estilos de aprendizaje y generen mayor motivación frente al aprendizaje de las matemáticas; de forma tal que se logre un mayor grado de desarrollo en el pensamiento matemático de sus estudiantes.

\section{Currículo}

El currículo comprende los propósitos de formación, los contenidos temáticos, las metodologías y las formas de evaluación. Es el mapa de carreteras que orienta las actividades de aprendizaje y evaluación a desarrollar en el aula. Cada profesor hace operativo el currículo en su día a día con sus estudiantes, dando respuesta personal al qué enseñar y cómo reflejarlo en su práctica. (Contreras Domingo, 1994)

Mucho se ha discutido sobre el diseño curricular de los cursos de formación matemática para estudiantes universitarios de disciplinas diferentes. Cabe formularse la pregunta: ¿Cuáles deben ser los criterios que guíen la selección de contenidos apropiados y necesarios para la formación de profesionales en pregrados como la Administración de Empresas? Al respecto, el trabajo de Rico (1998) es revelador: 
La funcionalidad del concepto de currículo se ha desarrollado mediante la búsqueda sistemática de niveles de reflexión, estableciendo componentes por cada nivel y relaciones entre las componentes de diferentes niveles. También se presentan los organizadores del currículo. El conocimiento didáctico sobre cada uno de los contenidos del currículo de matemáticas ha de quedar estructurado mediante la aportación que hacen cada uno de los organizadores a dicho contenido. El profesor de matemáticas necesita conocimientos sólidos sobre los fundamentos teóricos del currículo y sobre los principios para el diseño, desarrollo y evaluación de unidades didácticas de matemáticas. Se necesitan nuevas herramientas conceptuales con las cuales abordar las tareas de diseño, desarrollo y evaluación de unidades didácticas en el área de matemáticas. La caracterización operacional del currículo mediante objetivos, contenidos, metodología y evaluación no es inadecuada, sólo lo es su empleo en tareas de trabajo para el aula, sin criterios de referencia. (Rico, 1998)

En este orden de ideas, se plantea que el diseño curricular de los cursos de matemáticas para Administración debe incluir habilidades de dominio técnico y conceptual, enmarcadas en la usabilidad de los conceptos. (Blanco, 2014)

También se requiere incluir las bases para "la modelización cuantitativa de la realidad económica y empresarial, aprovechando las ventajas del enfoque matemático a la hora de la búsqueda del conocimiento económico." (Díaz \& Marbán, 2016)

Todo lo anterior, conduce a la necesidad de diseñar currículos con los niveles de conocimiento adecuados que permitan acercar al estudiante el estudio de las matemáticas contextualizada para la administración, de forma tal que incluya diferentes aplicaciones en temas financieros, logísticos o de marketing.

\section{Estrategias pedagógicas y diseño de actividades de aprendizaje}

$\mathrm{Si}$ se tienen en cuenta los factores mencionados con anterioridad, es claro que las estrategias pedagógicas que se adopten en las universidades deben definir cursos de acción para facilitar el aprendizaje, en este caso particular, de las matemáticas en pregrados de administración.

Estas acciones incluyen la adopción de metodologías innovadoras y el diseño de actividades de aprendizaje que influyan positivamente en los estudiantes.

Los estudios coinciden en la necesidad de implementar diseños instruccionales que no se centren solamente en el contenido, que fomenten en los estudiantes la construcción del significado de lo que está aprendiendo, que desarrollen el pensamiento crítico y la solución de problemas, y no sólo se centren en la memorización. (Camposeco, 2012) (Cardozo, Vanegas, \& Cerecedo, 2012)

Las estrategias que promueven la participación y el aprendizaje activo generan actitudes más positivas en los estudiantes y aumentan su motivación, pues "es necesario que los alumnos se sientan competentes para aprender, comprendan los contenidos que se trabajan en clase, cuenten con un ambiente en el aula que estimule y motive sus participaciones" (Veliz \& Pérez, 2004)

En una experiencia a reseñar al respecto, se diseñó un ambiente de aprendizaje semipresencial para estudiantes de primer semestre de Administración de la Universidad de Panamá sobre factorización. Los resultados evidenciaron que "se puede mejorar el proceso de aprendizaje de los estudiantes, si se diseñan estrategias que impliquen un participación más activa de ellos." (Alvarado, 2010) 
El aprendizaje colaborativo también ha mostrado generar impactos positivos en el aprendizaje de matemáticas. Un estudio realizado en la Universidad Nacional de Centro del Perú con estudiantes de tercer semestre de Administración señaló que "la estrategia activo colaborativo ha contribuido con el aprendizaje de los temas respecto a las aplicaciones de la derivada de una función. El buen uso de la estrategia activo colaborativo influye en forma significativa en el aprendizaje de análisis matemático." (Flores, Samaniego, \& Samaniego, 2014)

La metodología del Aprendizaje basado en problemas (ABP), se caracteriza por integrar el aprendizaje activo y el aprendizaje colaborativo, de acuerdo a los documentos del Tecnológico de Monterrey:

Es el modelo en el cual es el alumno quien busca el aprendizaje que considera necesario para resolver los problemas que se le plantean, los cuales conjugan aprendizaje de diferentes áreas de conocimiento. El método tiene implícito en su dinámica de trabajo el desarrollo de habilidades, actitudes y valores benéficos para la mejora personal y profesional del alumno.

El ABP puede ser usado como una estrategia general a lo largo del plan de estudios de una carrera profesional o bien ser implementado como una estrategia de trabajo a lo largo de un curso específico, e incluso como una técnica didáctica aplicada para la revisión de ciertos objetivos de aprendizaje de un curso. (ITESM, 2010)

El ABP ha sido empleado como técnica didáctica para el aprendizaje de matemáticas en administración en diferentes estudios y su impacto ha sido positivo en el aprendizaje y la motivación de los estudiantes. Por ejemplo, en las universidades Autónoma de Barcelona y Ramón Llull de la misma ciudad, se trabaja en el primer curso de matemáticas con los estudiantes de administración en el diseño de un presupuesto de ventas durante todo el semestre, con resultados positivos en el aprendizaje de los estudiantes. (Serrano, Bosch, \& Gascón, 2010)

Por su parte, el aprendizaje basado en proyectos también ha sido empleado en diferentes universidades latinoamericanas. Por ejemplo, se ha encontrado que fortalece la formulación de problemas matemáticos (Rodríguez, García, \& Lozano, 2015)

La tecnología es otra herramienta que puede generar ambientes de aprendizaje más favorables y cercanos a la experiencia de los estudiantes. Un estudio realizado en República Dominicana mostró que el uso de redes sociales generó una percepción más positiva de los estudiantes de la enseñanza de las matemáticas y de la resolución de problemas. (Cruz, 2016).

\section{Experiencias en el CESA}

A continuación se reseñan algunas estrategias y experiencias vinculadas con la promoción del aprendizaje de matemáticas a nivel de pregrado en el Colegio de Estudios Superiores de Administración (CESA). El CESA es una escuela de negocios situada en Bogotá, con una oferta académica de pregrado en Administración de Empresas y seis programas de postgrado, entre ellos cuatro Maestrías relacionadas con Administración, Marketing y Finanzas.

El pregrado en Administración de Empresas del CESA cuenta con más de mil estudiantes matriculados en la actualidad y es reconocido por su alta calidad en el entorno educativo y empresarial colombiano. En el plan de estudios existen cinco asignaturas de formación básica en temas de matemáticas y estadística: 
- Fundamentos matemáticos, que recoge los conceptos básicos del álgebra y el precálculo

- Cálculo 1, o cálculo diferencial

- Cálculo 2, o cálculo integral con algunos temas de cálculo multivariado

- Estadística Básica, donde se presentan temas de probabilidad y estadística descriptiva

- Estadística Aplicada, donde se trabajan los temas de estadística inferencial

El equipo de profesores que tiene a su cargo el desarrollo de estas asignaturas cuenta con un alto grado de compromiso con el aprendizaje de los estudiantes. Este grupo de docentes comparte las preocupaciones respecto a cómo incorporar nuevas estrategias pedagógicas que permitan dar mayor sentido al conocimiento en matemáticas para lograr aprendizajes más duraderos y un mayor desarrollo del pensamiento matemático en los estudiantes.

La enseñanza de matemáticas en el pregrado de Administración del Cesa no ha estado exenta de las dificultades señaladas en otras instituciones similares, respecto a las actitudes y motivación de los estudiantes, falta de hábitos de estudio, prácticas docentes con potencial de actualizarse y contextualizarse, un currículo poco flexible y poco contextualizado, bajo desarrollo del pensamiento matemático, entre otros.

Las estrategias que se han venido adoptando para mejorar la pedagogía de las matemáticas han incluido la creación del centro SUMA, la participación en capacitaciones de formación docente, la actualización de los contenidos y metodologías de las asignaturas y la inclusión de proyectos para promover el aprendizaje activo y colaborativo.

El área de matemáticas y estadística del CESA, con el respaldo institucional, ha creado el centro SUMA que busca el desarrollo del pensamiento matemático y estadístico (pensamiento numérico, variacional y aleatorio), aplicado a la Administración de Empresas. (CESA, 2018). SUMA fue concebido como un centro de apoyo al aprendizaje estudiantil, conforme a la definición propuesta por la Universidad de Chile, "orientado a brindar apoyo académico a los estudiantes, facilitando la nivelación de la brecha de contenidos que traen y posibilitando una mejor inserción en la vida universitaria." (Universidad de Chile, 2018)

Desde el inicio de su operación en 2015, SUMA ha contado con un equipo de docentes tutores dedicado a la atención de estudiantes que asisten para solucionar sus preguntas y realizar ejercicios complementarios que refuercen su aprendizaje en los temas de matemáticas y estadística. En la actualidad se cuenta con la información histórica de asistencia de estudiantes a SUMA y está en curso la realización de un estudio en el cual se espera medir cuál ha sido su impacto en el rendimiento académico de los estudiantes. También SUMA ha recopilado información sobre las actitudes de los estudiantes frente a las matemáticas, que espera difundir con los docentes próximamente.

SUMA también organizó una competencia matemática de carácter voluntario, cuyos premios para los primeros lugares les permitían obtener una bonificación pequeña en la calificación de su asignatura. La respuesta de los estudiantes fue masiva, en gran parte motivados por la calificación, pero de todas formas fue un espacio que les permitió ejercitar sus competencias matemáticas.

Por otra parte, el CESA ha creado un centro de Innovación pedagógica desde 2017, en el cual se ha configurado un equipo de personas que promueven la reflexión y actualización de las prácticas docentes. Este Centro, además de brindar asesorías individuales en didáctica a los docentes, programa capacitaciones y cursos de actualización a los docentes 
en temas de enseñanza, didáctica y evaluación. Los profesores del área de matemáticas han venido participando en estas capacitaciones.

En la actualidad se está adelantando el proceso de implementación de un nuevo plan de estudios en el pregrado de administración de empresas, en el cual se propuso una renovación curricular de los cursos tanto de matemáticas como de las demás áreas. En particular, los cursos de matemáticas se están diseñando en este momento con un enfoque aplicado a la administración, en el que se vinculen los conceptos matemáticos a problemas relacionados con temas de mercadeo, economía, finanzas, operaciones, logística y gestión. Una estrategia que se plantea es promover el aprendizaje por medio de proyectos de aplicación. El trabajo por proyectos ha mostrado en otras instituciones similares resultados favorables en cuanto al logro de resultados de aprendizaje de las matemáticas. (Masero, Camacho, M., \& Asián, 2017)

De todas maneras, desde hace algún tiempo algunos de los docentes vienen trabajando con estrategias de aprendizaje activo en temas puntuales de los cursos. El tránsito de las clases centradas en el contenido matemático a las clases centradas en el aprendizaje se viene dando gradualmente. Cada semestre se incluyen más aplicaciones relacionadas con la administración en los cursos de matemáticas, vinculadas con los conceptos matemáticos. También se ha llegado a un porcentaje asignado en la evaluación del 50\% para los problemas aplicados.

Vale la pena mencionar algunas de las actividades de aprendizaje que se han realizado en los cursos de matemáticas:

- Para evaluar los conceptos relacionados con funciones lineales o cuadráticas y sus gráficas, se asigna a los estudiantes de primer semestre un proyecto integrador con otra de sus asignaturas, Introducción a la administración. El trabajo grupal consiste en recopilar datos de una empresa a la cual se realiza un análisis desde su parte organizacional, lo cual se complementa con la modelación de sus ventas como funciones lineales o cuadráticas realizando una regresión lineal simple con Excel. Este proyecto es evaluado tanto por el profesor de Introducción como por el profesor de matemáticas. Permite a los estudiantes realizar su primera aproximación a la modelación matemática y reforzó la parte conceptual de funciones lineales y cuadráticas. En general, ha tenido una buena recepción por parte de los estudiantes y se afianzaron los conceptos matemáticos estudiados.

- Como parte del tema de ecuaciones de primer grado, se ha propuesto a los estudiantes el proyecto de planear una idea de negocio sencilla, para la cual se deben formular ecuaciones lineales de ingreso, costos y utilidad con datos lo más reales posibles para encontrar el punto de equilibrio. Este trabajo en grupos se presenta a sus compañeros de curso, quienes realizan preguntas y participan como coevaluadores. La motivación de los estudiantes es alta en esta actividad y se ha corroborado la incorporación del concepto en evaluaciones posteriores.

- Se vienen diseñando talleres con los estudiantes orientados a construir modelos matemáticos presentes con información real, por ejemplo con el Índice de Gini, en cálculo integral; con la función logística y la apropiación de nuevas tecnologías, en cálculo diferencial.

- Se ha propuesto a los estudiantes redactar el texto de un problema matemático desde una situación vivencial: la planeación de un almuerzo compartido, la organización de una fiesta o un viaje, el diseño de un empaque para un producto. 
Se ha venido trabajando sobre el supuesto de que los problemas y proyectos de aplicación en contexto plantean desafíos a los estudiantes que pueden elevar su motivación hacia el aprendizaje de las matemáticas. Estas acciones que se han venido implementando no han sido evaluadas todavía, por lo cual no se cuenta en la actualidad con indicadores del impacto que han tenido. Se tiene previsto realizar los estudios que permitan concluir cómo estos cambios en el currículo y las metodologías han influido en el aprendizaje de las matemáticas en los estudiantes del pregrado en Administración del CESA.

\section{A modo de conclusión}

Es responsabilidad de los docentes emplear continuar en la búsqueda de estrategias didácticas por encima del aprendizaje memorístico de fórmulas o la ejecución repetitiva de ejercicios matemáticos descontextualizados para lograr aprendizajes más significativos en sus estudiantes. Es también útil y necesario conocer más sobre las actitudes hacia la matemática de los estudiantes, para planear actividades que generen una mayor motivación hacia el aprendizaje.

Puede decirse que frente a la problemática en la enseñanza de las matemáticas en Administración de Empresas una alternativa valedera consiste en cambiar las estrategias didácticas y reorientarlas hacia el aprendizaje basado en problemas y en proyectos; así como el aprendizaje colaborativo y el aprendizaje activo, como lo señalan las experiencias presentadas de otras instituciones. Adicionalmente, estas estrategias pedagógicas deben propender por redireccionar la enseñanza de las matemáticas desde lo formal y lo teórico hacia lo aplicado, por medio de la actualización y contextualización del currículo.

En el CESA las estrategias para la enseñanza de las matemáticas para estudiantes de Administración se están centrando en el aprendizaje activo por medio de proyectos y problemas de aplicación.

Queda pendiente realizar y completar los estudios sobre lo que se viene trabajando en el CESA para reseñar en futuros documentos la medición del impacto de las acciones que se han venido tomando.

\section{Referencias}

Alvarado, D. (2010). Uso de unidades de autoinstrucción para la enseñanza de la factorización, en un curso de matemática i para administradores . En P. Lestón, Acta latinoamericana de matemática educativa (págs. 555-560). México DF: Comité Latinoamericano de matemática educativa.

Auzmendi, E. (1992). Las actitudes hacia la matemática/estadística en las enseñanzas medias y universitaria : características y medición. Bilbao: Ediciones el Mensajero.

Bandura. (1997). Self-efficacy. The excercise of control. Nueva York: Freeman.

Blanco, M. \&. (2014). Selección racional de contenidos matemáticos, estadísticos y econométricos en el grado en Administración y Dirección de Empresas en el Espacio Europeo de Educación Superior. Obtenido de Dialnet: https://dialnet.unirioja.es/

Cagliolo, L., Junco, C., \& Peccia, A. (2010). Investigación sobre las relaciones entre los estilos de aprendizaje y el resultado académico en las asignaturas elementos de matemática, introducción a la administración y analisis socio-economico . Revista Estilos de Aprendizaje, 23-33. 
Camposeco, F. d. (2012). La autoeficacia como variable en la motivación intrínseca y extrínseca en matemáticas a través de un criterio étnico. Obtenido de http://eprints.ucm.es/16670/

Cardozo, E., Vanegas, E., \& Cerecedo, M. (2012). Actitudes hacia las matemáticas de los estudiantes de posgrado en administración: un estudio diagnóstico . Educare, 237253.

CESA. (15 de 01 de 2018). Centro SUMA. Obtenido de http://www.cesa.edu.co/ElCesa/Suma.aspx

Contreras Domingo, J. (1994). Enseñanza, Didáctica y profesorado.Introducción crítica a la didáctica. Madrid: Akal.

Cruz, I. (2016). Percepciones de las redes sociales y su aplicación en la enseñanza de las matemáticas . Pixel-Bit. Revista de medios y educación, 165-186.

Díaz, F., \& Marbán, J. (2016). Análisis de la formación matemática en los actuales grados en administración y dirección de empresas. Obtenido de Repositorio Digital de documentos en educación matemática Uniandes: http://funes.uniandes.edu.co/8932/

Dorfer, C., \& Ulloa, S. (2016). Medición de la actitud hacia las matemáticas en estudiantes de licenciatura en administración: un estudio piloto . Vinculatégica, 1329-1348.

Flores, A., Samaniego, C., \& Samaniego, D. (2014). Estrategia activo colaborativo factor influyente en el aprendizaje de Análisis Matemático I, en estudiantes de Ciencias de la Administración. Prospectiva Universitaria, 57-66.

García de Pilo, L. (2010). La enseñanza de las matemáticas aplicadas a las carreras de administración y contaduría . Encuentro Educacional, 77-91.

Hernández, C., Ramírez, P., \& Rincón, G. (2013). Pensamiento matemático en estudiantes universitarios. Ecomatemático, 4-10.

ITESM, E. A. (2010). sitios.itesm.mx. Obtenido de Itesm.mx: http://sitios.itesm.mx/va/dide2/tecnicas_didacticas/abp/abp.pdf

Masero, I., Camacho, M., M., V., \& Asián, R. (2017). Aprendizaje activo de las Matemáticas para la Economía y Empresa: una experiencia docente. En R. Roig-Vila, Investigación en docencia universitaria. Diseñando el futuro a partir de la innovación educativa (págs. 626-633). Barcelona: Ediciones Octaedro.

Petriz, M., Barona, C., López, R., \& Quiroz, M. (2010). "Niveles de desempeño y actitudes hacia las matemáticas en estudiantes de la licenciatura en administración en una universidad estatal mexicana. Revista Mexicana de Investigación Educativa, 1223-1249.

Rico, L. (1998). Complejidad del currículo de matemáticas como herramienta profesional. Revista Latinoamericana de Investigación en Matemática Educativa, 22-39.

Rodríguez, L., García, L., \& Lozano, M. (2015). El método de proyecto para formulación de problemas matemáticos. Atenas, 100-112.

Rojas, C. \&. (2017). Motivación, ansiedad, confianza, agrado y utilidad. Los factores que explican la actitud hacia las matemáticas. INFAD, 527-539.

Serrano, L., Bosch, M., \& Gascón, J. (2010). Cómo hacer una previsión de ventas": propuesta de recorrido de estudio e investigación en un primer curso universitario de administración y dirección de empresas. IUFM. 
Silva Atacho, L. (2012). Fenómenos de la didáctica de la matemática en docentes de matemática del decanato de administración y contaduría de la ucla. Gestión y gerencia, 108-126.

Universidad de Chile. (15 de 01 de 2018). Centros para el aprendizaje estudiantil. Obtenido de http://www.uchile.cl/portal/presentacion/vicerrectoria-de-asuntos-estudiantiles-ycomunitarios/oficina-de-equidad-e-inclusion/iniciativas-destacadas/110453/centrospara-el-aprendizaje-estudiantil

Vargas, C. (2011). Resolución de problemas y pensamiento crítico:APRENC-Mates. propuesta de formación inicial de maestros. Unión. Revista interamericana de formación matemática, 117-128.

Veliz, M., \& Pérez, M. (2004). Las actitudes hacia la matemática y el rendimiento académico en alumnos de cálculo diferencial . Acta Latinoamericana de Matemática Educativa , 411-417.

Zubieta, C., \& Martínez, M. d. (2008). expectativas sobre matemáticas que tienen los estudiantes de la carrera de administración de la universidad autónoma metropolitana Azcapotzalco. Gestión y estrategia, 63-74. 